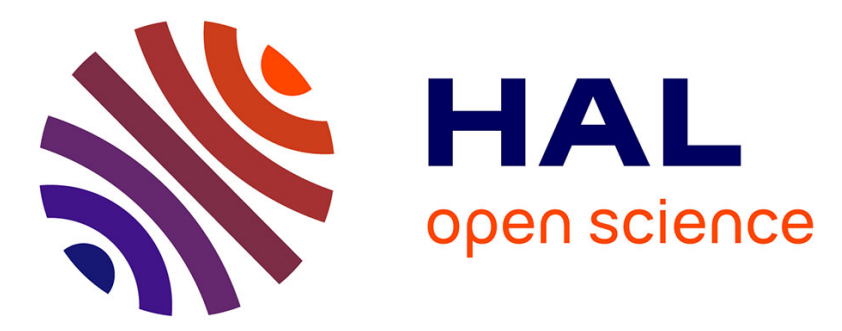

\title{
Human activities of daily living recognition using fuzzy logic for elderly home monitoring
}

\author{
Hamid Medjahed, Dan Istrate, Jérôme Boudy, Bernadette Dorizzi
}

\section{To cite this version:}

Hamid Medjahed, Dan Istrate, Jérôme Boudy, Bernadette Dorizzi. Human activities of daily living recognition using fuzzy logic for elderly home monitoring. FUZZ-IEEE 2009: IEEE International Conference on Fuzzy Systems, Aug 2009, Jeju Island, South Korea. pp.2001 - 2006, 10.1109/FUZZY.2009.5277257 . hal-01366500

\section{HAL Id: hal-01366500 https://hal.science/hal-01366500}

Submitted on 14 Sep 2016

HAL is a multi-disciplinary open access archive for the deposit and dissemination of scientific research documents, whether they are published or not. The documents may come from teaching and research institutions in France or abroad, or from public or private research centers.
L'archive ouverte pluridisciplinaire HAL, est destinée au dépôt et à la diffusion de documents scientifiques de niveau recherche, publiés ou non, émanant des établissements d'enseignement et de recherche français ou étrangers, des laboratoires publics ou privés. 


\title{
Human Activities of Daily Living Recognition Using Fuzzy Logic For Elderly Home Monitoring
}

\author{
Hamid Medjahed, Dan Istrate, Jerome Boudy and Bernadette Dorizzi
}

\begin{abstract}
Learning and recognizing human activities of daily living(ADL), is very useful and essential to build a pervasive home monitoring system. These monitoring technologies are indispensable for developing the next generation of smart houses. In this paper we describe a fuzzy logic system for recognizing activities in home environment using a set of sensors: physiological sensors (cardiac frequency, activity or agitation, posture and fall detection sensor), microphones, infrared sensors, debit sensors and state-change sensors. Motivated by the fact that Fuzzy controllers have been successfully embedded within billions of dollars in commercial products, plus the characteristic of data providing from each sensor, the fusion of the different sensors has been performed by using fuzzy logic. This fuzzy logic approach allowed us to recognize several Activities of Daily Living (ADLs) for Ubiquitous Healthcare.
\end{abstract}

\section{INTRODUCTION}

As people grow older, the current healthcare infrastructure in France is widely considered to be inadequate to meet the needs of this increasingly older population. Healthcare monitoring is a solution to deal with this problem and ensure the elderly to live safely and independently in their own home for as long as possible.

To improve and to develop this technological approach called Automatic health monitoring, the French National Agency for Research [1] has been funded and created various projects and consortia. Among those projects QuoVADis project $^{1}$, the one in which we propose a multimodal platform [2] for several uses and especially for recognizing the daily activities in order to provide proactive healthcare at home. Advances in miniature sensor and wireless technologies have resulted in interest to gather many sensors under our monitoring platform. It is based on three main subsystems, which have been technically validated from end to end, through their hardware and software, plus state change sensors for Home Appliances and water debit sensors.

The first subsystem is Anason [2] with its set of microphones that allow sound remote monitoring of the acoustical environment of the elderly. The second subsystem is RFpat [2], a wearable device fixed on the elderly person, that can measure physiological data like heart rate, activity, posture and an eventual fall done by the person. The last subsystem

Hamid Medjahed and Dan Istrate are with the LRIT laboratory, ESIGETEL, 1 rue du Port de Valvins, 77215, Avon-Fontainebleau cedex, France (phone: +33 (0)1 607270 51; fax: +33 (0)1 $6072 \quad 11$ 32; email: \{hamid.medjahed,dan.istrate $\} @$ esigetel.fr.

Hamid Medjahed, Jerome Boudy and Bernadette Dorizzi are with the Department of Electronics and Physics (EPH), Telecom Sud Paris, 9 rue Charles Fourier, 91011 Evry, France (phone:

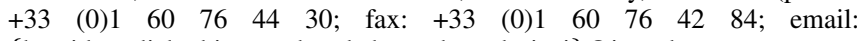
\{hamid.medjahed,jerome.boudy,bernadette.dorizzi\}@int-edu.eu.

${ }^{1}$ http://quovadis.ibisc.univ-evry.fr/ is a set of infrared sensors called Gardien [2], that detects the presence of the person in a given part and also the person's standing posture.

In order to maximize correct recognition of the various ADLs like sleeping, cleaning, bathing etc..., data fusion over the different sensors types is studied. The area of data fusion has generated great interest among researchers in several science disciplines and engineering domains. We have identified two major classes of fusion techniques: (1) Those that are based on probabilistic models (such as Bayesian reasoning [3] and the geometric decision reasoning like Mahanalobis distance), but their performances are limited when the data are heteregeneous and insufficient for the correct statistical modeling of classes, therefore the model is uncontrollable. (2) Those based on connectionist models (such as neural networks MLP [4] and SVM [5]) which are very powerful because they can model the strong nonlinearity of data but with complex architecture, thus lack of intelligibility. Based on those facts and considering the complexity of the data to process (audio, physiologic and multisensory measurements) plus the lack of training sets that reflect activities of daily living, fuzzy logic has been found useful to be the decision module of our multimodal ADLs recognition system. Fuzzy logic can gather performance and intelligibility and it deals with imprecision and uncertainty. It has a background application history to clinical problems including use in automated diagnosis [6], control systems [7], image processing [8] and pattern recognition [9]. For medical experts is more easier to map their knowledge onto fuzzy relationships than to manipulate complex probabilistic tools. In this paper, a novel and robust ADL recognition approach based on fuzzy logic is proposed. By combining a set of sensors, the recognition rate of ADL can be improved significantly in a real usage environment.

\section{State Of The Art And Research Motivation}

Everyday life activities in the home split into two categories. Some activities show the motion of the human body and its structure. Examples are walking, running, standing up, setting down, laying and exercising. These activities may be most easily recognized using sensors that are placed on the body (e.g. [10][11][12]). A second class of activities is recognized by identifying or looking for patterns in how people move things. In this work we focus on some activities identification belong to these both categories by using fuzzy logic. The use of fuzzy logic in our platform is motivated by two main raisons from a global point of view: 
- Firstly the characteristic of data to merge which are measurements obtained from different sensors, thus they could be imprecise and imperfect.

- Secondly, the history of fuzzy logic proves that it is used in many cases which are necessary for pattern recognition applications.

\section{A. Fuzzy Logic And Patterns Recognition Systems}

We use fuzzy logic for ADLs recognition, which is a fuzzy set theory, introduced by Lotfi A. Zadeh [13] in 1965, it is an extension of classical set theory. Historically, this was closely related to the concept of fuzzy measure, proposed just after by Sugeno [14]. Similar attempts at proposing fuzzy concept were also made at the same time by Shafer (evidence theory [15]) and Shackle (surprise theory [16]). Since that time, fuzzy logic has been more studied, and several applications were developed, essentially in Japan. The use of fuzzy sets can be done mainly at two levels:

a) Attributes representation: It may happen that data are uncomplete or noisy, unreliable, or some attributes are difficult to measure accurately or difficult to quantify numerically. At that time, it is natural to use fuzzy sets to describe the value of these parameters. The attributes are linguistic variables, whose values are built with adjectives and adverbs of language: large, small, medium etc...and as an illustrating example, we found the recognition system proposed by Mandal et al. [17]. Some methods are based on a discretization of the attributes space defined as language. Thus a numerical scale of length will be replaced by a set of fuzzy labels, for example (very small, small, medium, large, extra large), and any measure of length, even numerical is converted on this scale. The underlying idea is to work with the maximum granularity, i.e. the minimum accuracy.

b) Class representation: Groups do not create a clear partition of the data space, but a fuzzy partition where recovery is allowed will be better adapted. A significant number of fuzzy patterns recognition methods, are just an extension of traditional methods based on the idea of fuzzy partition for example the fuzzy c-means algorithm [18]. Historically, the idea of fuzzy partition was first proposed by Ruspini [19] in 1969. Rather than creating new methods of fusion and patterns recognition based on entirely different approaches, fuzzy logic fit naturally in the expression of the problem of classification, and tend to make a generalization of the classification methods that already exist. Taking onto account the four steps of a recognition system proposed by Bezdek et Pal [20], fuzzy logic is very useful for these steps.

- Data description: Fuzzy logic is used to descript syntactic data [21], numerical and Contextual data, conceptual or rules based data [22] which is the most significant contribution for the data description.

- Analysis of discriminate parameters: In image processing, there are many techniques based on fuzzy logic for segmentation, detection, contrast enhancement [23] and extraction [24].

- Clustering algorithms: The aim of these algorithms is to label a set of data into $\mathrm{C}$ groups, so that obtained groups contain the most possible similar individuals. Fuzzy c-mean algorithm and fuzzy ISODATA [25] algorithm are the the better known in this category.

- Design of the discriminator: The discriminator is designed to produce a fuzzy partition or a clear one, describing the data. This partition corresponds to a set of classes. Indeed the fuzzy ISODATA algorithm will be adapted for this step.

\section{B. How To Do Fuzzy Logic}

Fuzzy logic is a powerful framework for performing automated reasoning. It reflects human reasoning based on inaccurate or incomplete data. It uses the concept of partial membership, each element belongs partially or gradually to fuzzy sets that have been already defined. In contrast to classical logic where the membership function $u(x)$ of an element $x$ belonging to a set $A$ could take only two values: $u_{A}(x)=1$ if $x \in A$ or $u_{A}(x)=0$ if $x \notin A$, fuzzy logic introduces the concept of membership degree of an element $x$ to a set $A$ and $u_{A}(x) \in[0,1]$, here we speak about truth value. Figure 1 shows the main fuzzy inference system steps

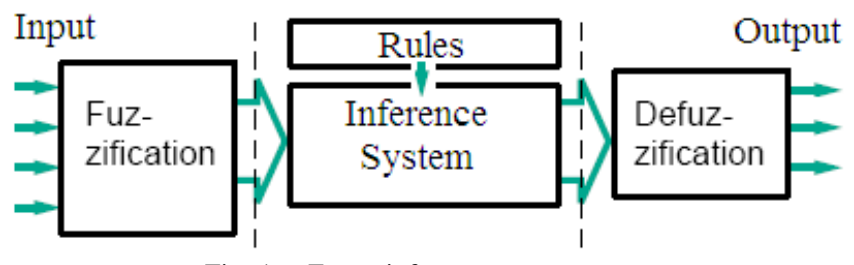

Fig. 1. Fuzzy inference system steps.

that are used our ADLs recognition system which are:

1) Fuzzification: First step in fuzzy logic is to convert the measured data into a set of fuzzy variables. It is done by giving value (these will be our variables) to each of a membership functions set. Membership functions take different shape. A Triangular membership function with straight lines can formally be defined as follows:

$$
\Lambda(x, a, b, c)=\left\{\begin{array}{rc}
0, & x \leq a \\
(x-a) /(b-a), & a \leq x \leq b \\
(c-x) /(c-b), & b \leq x \leq c \\
0, & x \geq c
\end{array}\right.
$$

Trapezoidal Function Furnished in the equation 2.

$$
f(x, a, b, c)=\left\{\begin{array}{rc}
0, & x \leq a \\
(x-a) /(b-a), & a \leq x \leq b \\
1, & b \leq x \leq c \\
(d-x) /(d-c), & c \leq x \leq d \\
0, & x \geq d
\end{array}\right.
$$

A Gaussian membership function with the parameters $\mathrm{m}$ and $\sigma$ to control the center and width of the membership function is defined by

$$
G(x, m, \sigma)=e^{\frac{-(x-m)^{2}}{2 \sigma^{2}}}
$$


The generalized Bell function depends on three parameters $\mathrm{a}, \mathrm{b}$, and $\mathrm{c}$ is given by

$$
f(x, a, b, c)=\frac{1}{1+\|(x-c) / a\|^{2 b}}
$$

There are also other membership functions like sigmoidally shaped function, single function etc... The choice of the function shape is iteratively determinate, according to type of data and taking into account the experimental results.

2) Fuzzy rules and inference system: The fuzzy inference system uses fuzzy equivalents of logical AND, OR and NOT operations to build up fuzzy logic rules. An inference engine operates on rules that are structured in an IF-THEN format. The IF part of the rule is called the antecedent, while the THEN part of the rule is called the consequent. Rules are constructed from linguistic variables. These variables take on the fuzzy values or fuzzy terms that are represented as words and modeled as fuzzy subsets of an appropriate domain. There are several types of fuzzy rules, we mention only the two mains used in our system:

- Mamdani rules [26] which is of the form: If $x_{1}$ is $A_{1}$ and $x_{2}$ is $A_{2}$ and...and $x_{p}$ is $A_{p}$ Then $y_{1}$ is $C_{1}$ and $y_{2}$ is $C_{2}$ and....and $y_{p}$ is $C_{p}$. Where $A_{i}$ and $C_{i}$ are fuzzy sets that define the partition space. The conclusion of a Mamdani rule is a fuzzy set. It uses the algebraic product and the maximum as Tnorm and S-norm respectively, but there are many variations by using other operators.

- Takagi/Sugeno rules [26]: If $x_{1}$ is $A_{1}$ and $x_{2}$ is $A_{2}$ and...and $x_{p}$ is $A_{p}$ Then $y=b_{0}+b_{1} x_{1}+b_{2} x_{2}+$ $\ldots+b_{p} x_{p}$. In the Sugeno model the conclusion is numerical. The rules aggregation is in fact the weighted sum of rules outputs.

3) Defuzzification: The last step of a fuzzy logic system consists in turning the fuzzy variables generated by the fuzzy logic rules into real value again which can then be used to perform some action. There are different defuzzification methods, in our platform decision module we could use Centroid of area (COA), Bisector of area (BOA), Mean of Maximum (MOM), Smallest of Maximum (SOM) and Largest of Maximum (LOM). Equations 5, 6, 7 and 8 illustrate them.

$$
\begin{gathered}
Z_{C O A}=\frac{\sum_{i=1}^{n} u_{A}\left(x_{i}\right) x_{i}}{\sum_{i=1}^{n} u_{A}\left(x_{i}\right)} \\
Z_{B O A}=x_{M} ; \sum_{i=1}^{M} u_{A}\left(x_{i}\right)=\sum_{j=M+1}^{n} u_{A}\left(x_{j}\right) \\
Z_{M O M}=\frac{\sum_{i=1}^{N} x_{i}^{*}}{N} \\
Z_{S O M}=\min \left(x_{i}^{*}\right) \text { and } Z_{L O M}=\max \left(x_{i}^{*}\right)
\end{gathered}
$$

where $x_{i}^{*}(i=1,2, \ldots, N)$ reach the maximal values of $u_{A}(x)$.

\section{FuZZY LOGIC ACTIVITy Detection ApPROACH}

The main advantages of using fuzzy logic system are the simplicity of the approach and the capacity of dealing with the complex data acquired from the different sensors. Fuzzy set theory offers a convenient way to do all possible combinations with these sensors. Fuzzy set theory is used in this system to monitor and to recognize the activities of people within the environment in order to timely provide support for safety, comfort, and convenience. Automatic health monitoring is predominantly composed of location and activity information. Abnormality also could be indicated by the lack of an activity or a abnormal activity detection which will cause or rise the home anxiety. Table I lists exactly what we wish to automatically recognize.

TABLE I

LIST OF ADLS RECOGNITION BY OUR PLATFORM.

\begin{tabular}{c|c}
\hline \hline ADLs of using devices & ADLs of Human body motion \\
\hline getting up,toilete, bathing, & sleeping, walking, standing up, \\
soing out of home, enter home & exercising \\
washing dishes,doing laundry, resting & \\
washing hands, watching TV, \\
listening radio, cleaning \\
talking on telephone,cooking & \\
\hline \hline
\end{tabular}

The first step for developing this approach is the fuzzification of system outputs and inputs obtained from each sensor and subsystem.

TABLE II

FUZZY SETS DEFINED FOR THE ANASON CLASSIFICATION INPUT.

\begin{tabular}{c|c}
\hline \hline membership function & Composition \\
\hline Human sound & $\begin{array}{c}\text { snoring, yawn, sneezing, } \\
\text { cough, cry, scream, laught }\end{array}$ \\
\hline Speech & key words and expressions \\
\hline Tultimedia sound & door claping, door knob \\
Door sound & key ring \\
\hline Water sound & water flushing, water in washbasin, \\
& coffee filter \\
\hline Ringtone & $\begin{array}{c}\text { telephone ring, bell door, } \\
\text { alarm, alarm clock }\end{array}$ \\
\hline Object sound & chair, table, \\
& tear-turn paper, step foot \\
\hline Machine sound & $\begin{array}{c}\text { coffee machine, dishwasher, } \\
\text { electrical shaver,microwave, } \\
\text { vaccum cleaner, washing machine, } \\
\text { air conditioner }\end{array}$ \\
& $\begin{array}{c}\text { glass vs glass, glass wood, } \\
\text { plastic vs plastic, plastic vs wood, } \\
\text { spoon vs table...etc }\end{array}$ \\
\hline \hline
\end{tabular}

From Anason subsystem three inputs are built. The first one is the sound environment classification, all sound class and expressions detected are labeled on a numerical scale according to their source. Nine membership functions are set up in this numerical scale according to sound sources as it is in table II. Two other inputs are associated to each SNR calculated on each microphone(two microphones are used in 
the current application), and these inputs are split into three fuzzy levels: low, medium and high.

RFpat produce five inputs; Heart rate for which three fuzzy levels are specified normal, low and high; Activity which has four fuzzy sets: immobile, rest, normal and agitation; Posture is represented by two membership functions standing up / setting down and lying; Fall and call have also two fuzzy levels: Fall/Call and No Fall/Call. The defined area of each membership function associated to heart rate or activity is adapted to each monitored elderly person. In our application we use only posture, and activity inputs.

For each infrared sensor $C_{i}$ a counter of motion detection with three fuzzy levels (low, medium, high) is associated, and a global one for all infrared sensors.

The time input has five membership functions morning, noon, afternoon, evening and night which are also adapted to patient habits.

For each main machine in the house a change state sensor $\mathrm{S}_{\text {device'sname }}$ is associated. It has two membership functions turn on and turn off. One debit sensor for water is included in our application. Three membership functions characterize this sensor, low, medium and high.

The output of our fuzzy logic ADL recognition contains somme activities which are selected from the table I. They are Sleeping (S), Getting up (GU), Toileting (T), Bathing (B), Washing hands (WH), Whashing dishs (WD), Doing laundry (DL), Cleaning (CL), Going out of home (GO), Enter home (EH), Walking (W), Standing up (SU), Setting down (SD),Laying (L), Resting (R), Watching TV (WT) and Talking on telephone (TT). These membership functions are ordered, firsly according to the area where they maybe occur and secondly according to the degree of similarity between them.

Figure 2 shows the in-home sensors disposal and the repartition of the home into areas in order to evaluate and to supervise the ADL recognition system. Microphones have been calibrated using a standard level sinusoidal signal with a frequency of $1 \mathrm{KHz}$. Infrared sensors are also calibrated and for each one a specific monitoring zone is delimited. The next step of our fuzzy logic approach is the fuzzy inference engine which is formulated by a set of fuzzy IF-THEN rules.

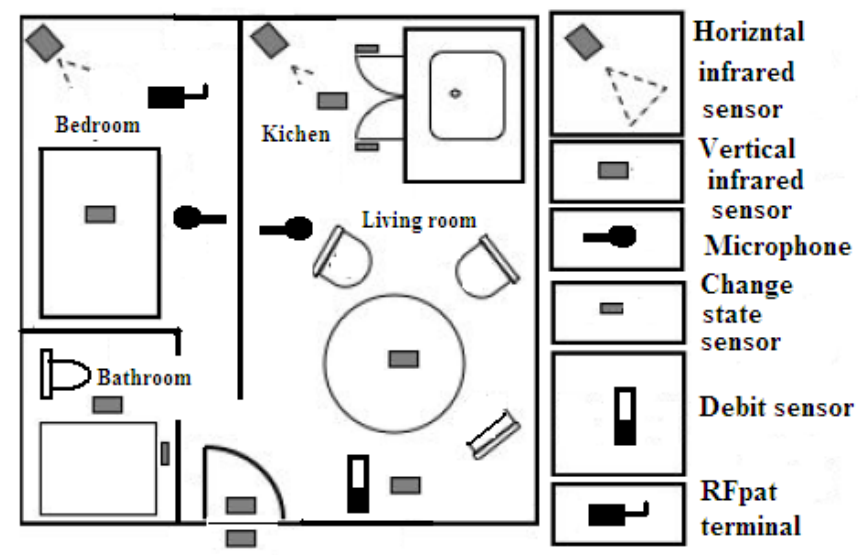

Fig. 2. Overview of a typically instrumented in-home.
This second stage uses domain expert knowledge regarding activities to produce a confidence in the occurrence of an activity. Rules allow the recognition of common performances of an activity, as well as the ability to model special cases. An example fuzzy rule for alarm detection is:

- If (Anason is Machine sound) and (Activity is motion) and $\left(\mathrm{C}_{\text {Overall }}\right.$ is high) and ( $\mathrm{CB}$ is high) and (C5 is high) and ( $\mathrm{S}_{\text {vaccum }}$ is turn on) Then (ADL is Cleaning).

A confidence factor is accorded to each rule and in order to aggregate these rules we have the choice between Mamdani or Sugeno approaches available under our fuzzy logic component. After rules aggregation the defuzzification is performed by the centroid of area for the ADL output.

\section{IMPLEMENTATION}

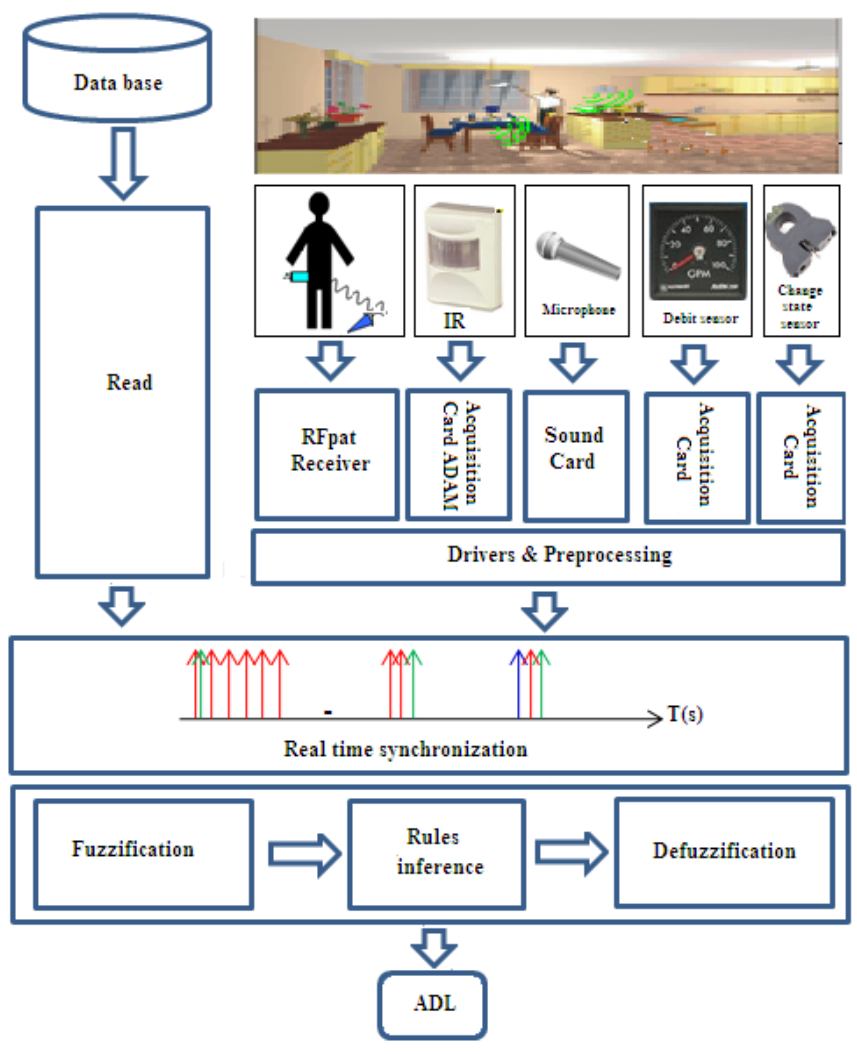

Fig. 3. Software architecture of ADL recognition platform.

Figure 3 provides a synoptic block-diagram scheme of the software architecture of the ADL recognition platform, it is implemented under Labwindows CVI and C++ software. It is developed in a form of design component. We can distinguish three main components, the acquisition module, the synchronization module and the fuzzy inference component. It can run off-line by reading data from a data base or online by processing in real time data acquired via the acquisition module.

To avoid the loss of data, a real time module with two multithreading tasks is integrated in the synchronization component. The platform is now synchronized on Gardien subsystem because of his smallest sampling rate $(2 \mathrm{~Hz})$ and 


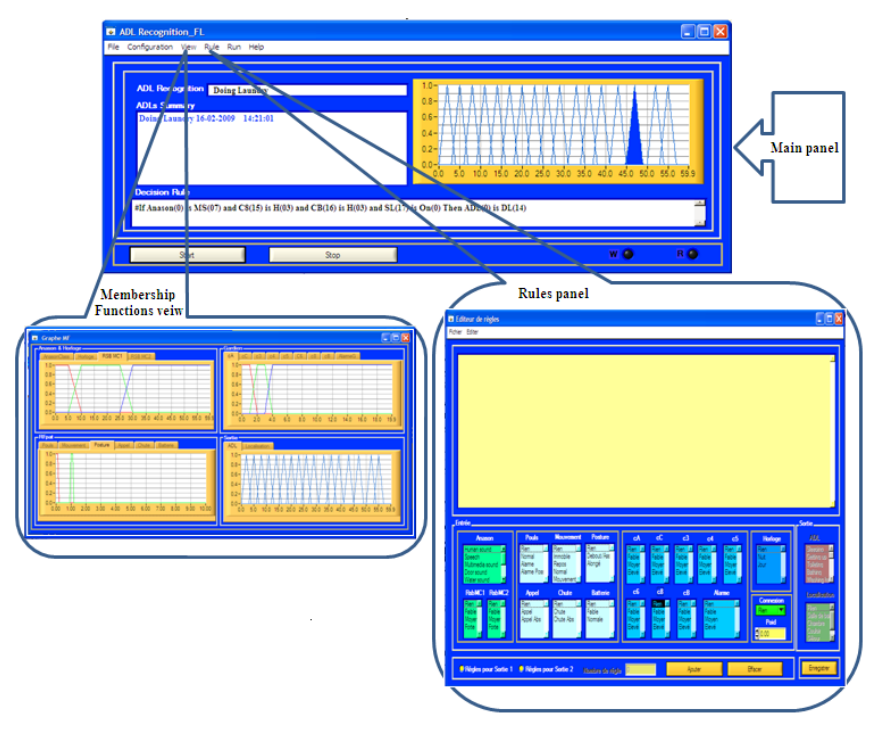

Fig. 4. The Software of ADL recognition platform.

periodicity. Indeed in some situations the Rfpat system may be not used by the elderly person, namely if no recommandations relative to its cardiac watch or a particular risk of fall are given by the Doctor.

We have developed a software with Fuzzy tools which allow the easy configuration of input intervals of fuzzyfication, the writing of fuzzy rules and the configuration of the defuzzyfication method. The general interface of the system allows to build up membership functions of inputs and outputs and displaying them. We could also write rules on text file by using a specific language, that we have developed, understandable by our system as it is in figure 4. This framework also allows for rules to be added, deleted, or modified to fit each particular resident based on knowledge about their typical daily activities, physical status, cognitive status, and age. The software implementation is validated with many experimental tests. The results and the rules which produced them will be displayed on the main panel.

\section{ExPERIMENTAL Setup}

The proposed method was experimentally achieved on a simulated data in order to demonstrate its effectiveness. This simulation gaves very promising results for the ADL recognition.

Figure 5 shows results for a stream of a simulated data. This fist study was devoted to the evaluation of the system by taking into account rules used in this fuzzy inference system.

The used strategy consisted in realizing several tests with different combination rules, and based on obtained results one rule is added to the selected set of rules in order to get the missed detection. With this strategy good results are reached for the ADL output (about $97 \%$ of good ADL detection).

The simulation described here is preliminary but demonstrates that ubiquitous, simple sensor devices can be used to recognize activities of daily living from real homes. The system can be easily retrofitted in existing home environments with no major modifications or damage.

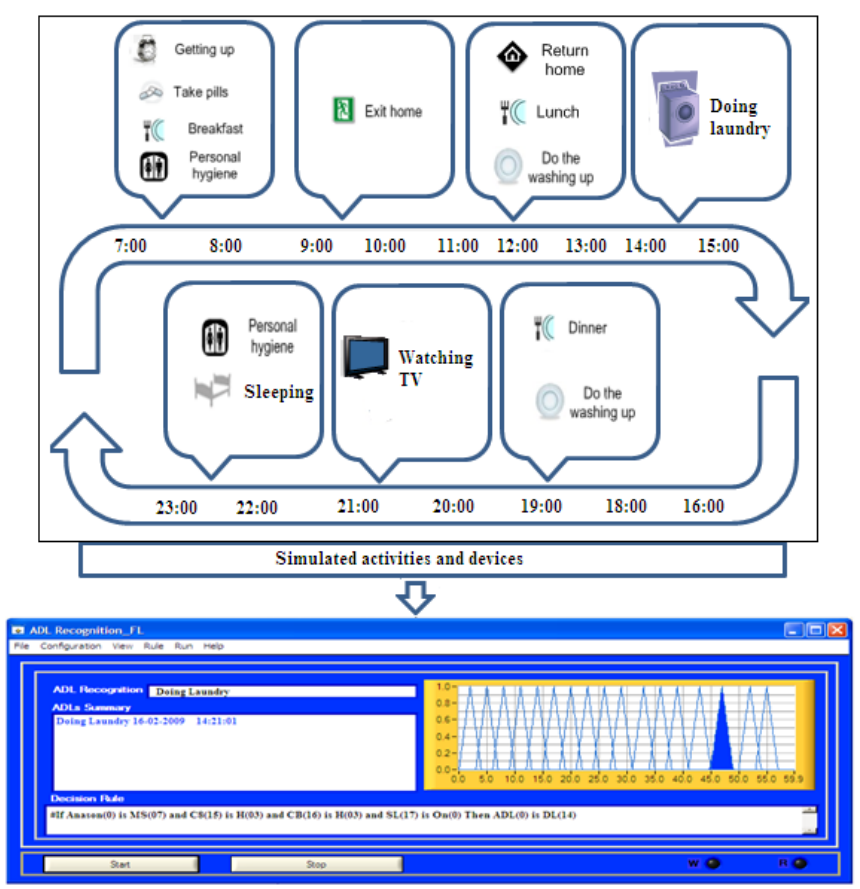

Fig. 5. ADL recognition experiment for a simulated stream data.

\section{CONCLUSIONS}

We have presented our ongoing research on recognition of activities in daily living. Our approach is based on fuzzy logic provide robust and high accuracy recognition rate on the simulated data. We are currently testing this approach on real time data in a real environment with strong confidence. It will be very useful for assisting elderly persons in place. The next objective of this research is to use these identification activities for building a model for measuring the home anxiety, that increases or decreases according to the detection activity and the state of each device in the home.

\section{ACKNOWLEDGMENT}

This research work was made in the framework of QuoVADis project. The authors gratefully acknowledge the contribution of French National Research Association (ANR) to QuoVADis Project.

\section{REFERENCES}

[1] www.agence-nationale-recherche.fr

[2] H. Medjahed, et al, "A Multimodal Platform for Database Recording and Elderly People Monitoring," Proc. BIOSIGNALS, Funchal-Madeira, Portugal, 28-31 January 2008, pp. 385-392.

[3] R. Cowell, A. Dawid, S. Lauritzen, and D. Spiegelhalter, Probabilistic Networks and Expert Systems, 1999, ISBN : 0-387-98767-3.

[4] G. Dreyfus, J.M Martinez, M. Samuelides, M. Gordon, F. Badran, S. Thiria and L. Hrault, Rseaux de neurones. Mthodologie et applications, Eyrolles, 2002.

[5] C. J. C. Burges, A tutorial on SVM for Pattern Recognition. Data Mining and Knowledge Discovery, volume 2, 1998, pp. 121-167.

[6] K. P. Adlassnig, Fuzzy set theory in medical diagnosis, IEEE Tr. On Syst.,Man, and Cybernetics, March/April 1986,pp. 260-265.

[7] D. Mason, D. Linkens and N. Edwards, Self-learning fuzzy logic control in medicine, Proc. AIME'97, (E. Keravnou et al., eds.), Lecture Notes in Artificial Intelligence 1211, Springer-Verlag, Berlin 1997, pp. 300-303.

[8] A. Lalande, et al., Automatic detection of cardiac contours on MR images using fuzzy logic and dynamic programming, Proc. AMIA Ann. Fall Symp. 1997, pp. 474-478. 
[9] G. Zahlmann, M. Scherf and A. Wegner, A neurofuzzy classifier for a knowledge-based glaucoma monitor, Proc. AIME'97, (E. Keravnou et al., eds.), Lecture Notes in Artificial Intelligence 1211, Springer-Verlag, Berlin 1997, pp. 273-284.

[10] M. Makikawa and H. Iizumi, "Development of an ambulatory physical activity monitoring device and its application for categorization of actions in daily life," in MEDINFO, pp. 747-750, 1995.

[11] J. Himberg J. Mantyjarvi and T. Seppanen, Recognizing human motion with multiple acceleration sensors,'IEEE Transactions on Systems, Man, and Cybernetics, pp. 747-52, IEEE Press, 2001.

[12] S.W Lee and K. Mase, "Activity and location recognition using wearable sensors," IEEE Pervasive Computing, 1(3):2432, 2002.

[13] L.A. Zadeh, "Fuzzy sets as a basis for theory of possibility, Fuzzy Set Systems," pp. 3-28, 1978.

[14] M. Sugeno, "Theory of fuzzy integrals and its applications," Doct. Thesis, Tokyo Institute of technology 1974.

[15] G. Shafer, "A Mathematical Theory of Evidence," Princeton Univ. Press 1979.

[16] G.L. Shackle, "Decision, Order and Time in Human Affairs," Cambridge Univ. Press

[17] D.P. Mandal, C. A. Murthy, and S. K. Pal, "Formulation of a multivalued recognition system," IEEE Transactions on Systems, Man, and Cybernetics, 22:607-6201992.

[18] W. Pedrycz, "Fuzzy sets in pattern recognition: methodology and methods," Pattern Recognition, 23(1/2):121-146, 1990.

[19] E. H. Ruspini, "A new approach to clustering," Inform, Control, 15(1):22-32, 1969.

[20] J. C. Bezdek and S. K. Pal, "Fuzzy Models for Pattern Recognition," IEEE Press, 1992

[21] M. Mizumoto, J. Toyoda and K. Tanaka, "General formulation of formal grammars," Info Sci., 4:87-100, 1972.

[22] Y. H. Pao, "Adaptive Pattern Recognition and Neural Networks," Addison-Wesley, 1989.

[23] J.M. Keller, R. Krishnapuram, "Fuzzy set methods in computer vision," In R.R. Yager and L.A. Zadeh, editors, An Introduction to Fuzzy logic Applications in Intelligent Systems Kluwer Academic, pp. 121-145, 1992.

[24] S.K. Pal and B. Chakraborty, "Fuzzy set theoretic measure for automatic feature evaluation," IEEE Transactions on Systems, Man, and Cybernetics, 16:754-760, 1986.

[25] J.C. Dunn, "A fuzzy relative of the isodata process and its use in detecting compact well-seperated clusters," IEEE Transactions on Systems, Man, and Cybernetics, pp. 32-57, 1973.

[26] J.-S. R. Jang, C. T Sun and E. Mizutani, "Neuro-Fuzzy and Soft Computing : A Computational Approach to Learning and Machine Intelligence," Prentice Hall Upper Saddle River, NJ 1997. 